\title{
Évaluer pour apprendre
}

L'évaluation constitue toujours un défi majeur du processus pédagogique. Dans quelle mesure les apprenants apprennent-ils? Quels moyens sont à privilégier pour évaluer des apprentissages? Ces moyens reflètent-ils vraiment les apprentissages réalisés? Quelle est la place des technologies de l'information dans l'évaluation? À Montréal, du 29 au 31 mai 2019, le $8^{\mathrm{e}}$ Forum international francophone de pédagogie des sciences de la santé (FIFPSS) réunira plusieurs experts en évaluation afin d'explorer le thème « Évaluer pour apprendre », à savoir les liens entre l'évaluation et les apprentissages. Ce thème particulier se situe au cœur des nouvelles approches pédagogiques issues de la psychologie cognitive et du socio-constructivisme où l'évaluation est intégrée au processus d'apprentissage.

Six conférenciers originaires de la francophonie mondiale partageront leurs savoirs, leurs pratiques et leurs recherches en évaluation. Il sera entre autre question de l'évaluation comme élément de motivation pour apprendre, de la rétroaction, du compagnonnage cognitif et de l'évaluation longitudinale des apprentissages. On y abordera aussi l'évaluation qui vise l'amélioration des pratiques professionnelles.

Par ailleurs, le Forum se veut un événement qui rassemble la communauté francophone de formateurs en sciences de la santé. C'est pourquoi, élargissant le thème central, le comité scientifique a choisi un éventail d'ateliers, de présentations libres et d'affiches qui reflètent la richesse des réflexions et des expériences de cette communauté. Le lecteur trouvera dans les pages de cette édition spéciale de Pédagogie Médicale les résumés des activités de ce nouveau Forum.

Au plaisir de vous accueillir à Montréal.

Présidente, comité scientifique FIFPSS Johanne Goudreau, IP, PhD

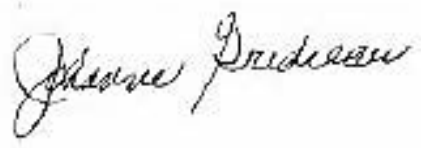

Présidente du FIFPSS Nicole Parent, PhD

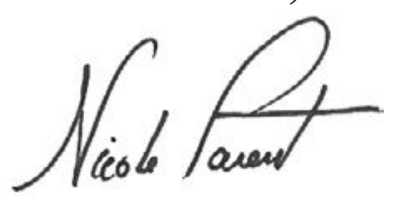

